\title{
In vitro comparative optical bench analysis of a spherical and aspheric optic design of the same IOL model
}

\author{
Tamer Tandogan ${ }^{1 *}$ (D), Gerd U. Auffarth ${ }^{1}$, Chul Y. Choi ${ }^{1,2}$, Stephanie Liebing ${ }^{1}$, Christian Mayer ${ }^{3}$ and Ramin Khoramnia ${ }^{1}$
}

\begin{abstract}
Background: To analyse objective optical properties of the spherical and aspheric design of the same intraocular lens (IOL) model using optical bench analysis.

Methods: This study entailed a comparative analysis of 10 spherical C-flex $570 \mathrm{C}$ and 10 aspheric C-flex $970 \mathrm{C} \mathrm{IOLS}$ (Rayner Intraocular Lenses Ltd., Hove, UK) of 26 diopters [D] using an optical bench (OptiSpheric, Trioptics, Germany). In all lenses, we evaluated the modulation transfer function (MTF) at $50 \mathrm{lp} / \mathrm{mm}$ and $100 \mathrm{lp} / \mathrm{mm}$ and the Strehl Ratio using a 3-mm (photopic) and 4.5-mm (mesopic) aperture.

Results: At $50 \mathrm{lp} / \mathrm{mm}$, the MTF values were 0.713/0.805 (C-flex 570 C/C-flex 970 C) for a 3-mm aperture and 0.294/ 0.591 for a $4.5-\mathrm{mm}$ aperture. At $100 \mathrm{lp} / \mathrm{mm}$, the MTF values were $0.524 / 0.634$ for a $3-\mathrm{mm}$ aperture and 0.198/0.344 for a 4.5-mm aperture. The Strehl Ratio was 0.806/0.925 and 0.237/0.479 for a 3-mm and 4.5-mm aperture respectively. A Mann-Whitney $U$ test revealed all intergroup differences to be statistically significant $(p<0.01)$.

Conclusion: The aspheric IOL design achieved higher MTF values than the spherical design of the same IOL for both apertures. Moreover, the differences between the two designs of the IOL were more prominent for larger apertures. This suggests that the evaluated IOL provides enhanced optical quality to patients with larger pupils or working under mesopic conditions.
\end{abstract}

Keywords: Aspheric IOL, MTF, Optical quality, Strehl ratio, Spherical IOL

\section{Background}

Irrespective of age, the regular healthy human cornea is attributable with a positive spherical aberration (SA). In young eyes, the negative SA of the crystalline lens usually counterbalances the cornea's positive SA, resulting in low SA overall. But with aging, the lens's SA changes from negative to positive, adding to the stable positive SA of the cornea and thereby decreasing the patient's overall optical quality [1-3].

Conventionally, standard intraocular lenses (IOLs) used for cataract surgery or refractive lens exchange feature a spherical design. Similar to the aged natural lens, these spherical IOLs have a positive SA, which adds

\footnotetext{
* Correspondence: tamer.tandogan@med.uni-heidelberg.de

'David J Apple International Laboratory for Ocular Pathology and

International Vision Correction Research Centre (IVCRC), Department of

Ophthalmology, University of Heidelberg, Im Neuenheimer Feld 400,

Heidelberg 69120, Germany

Full list of author information is available at the end of the article
}

to the cornea's positive SA. This results in reduced optical quality when compared to the juvenile natural lens. By comparison, implanting an aspheric IOL with a specific SA can lead to a significantly improved quality of vision for the patient, by modifying the overall ocular SA. These effects become more prominent with increasing pupil size, as SA is strongly dependent on aperture size. Numerous studies have proven the positive effect of aspheric IOLs on patients' visual functions [4-7].

It is important to note, however, that other studies have demonstrated that there can be also very little to no significant difference between spherical and aspheric IOLs of similar or equivalent models $[8,9]$. Several concepts of aspheric IOLs are available, such as IOLs with varying negative values of SA, "aberration neutral IOLs" $(\mathrm{SA}=0 \mu \mathrm{m})$, and IOLs with a progressive change of SA from centre to periphery [10-13]. 
There are several external factors that can influence postoperative ocular SA, such as the change in corneal SA induced by surgical corneal incisions [14]. Therefore, it is of significant importance that aspheric IOLs are manufactured with little tolerance to deviations from the intended SA in order to avoid an additional source of error to the sum of residual SA.

Our study is intended to determine differences in some of the optical properties of the "aberration-neutral" aspheric IOL and the spherical IOL. To enable a meaningful comparison, we selected IOLs that shared the same manufacturer, material and dioptric power; the only variant was SA. We analysed the optical properties of the IOL models on the optical bench according to international standardised testing methods to provide objective data on established optical property parameters, such as the Modulation Transfer Function (MTF) and the Strehl Ratio [15]. All analyses were performed for two different aperture sizes $(3 \mathrm{~mm}$ and $4.5 \mathrm{~mm}$ ) to represent photopic and mesopic surrounding conditions.

In summary, this study is intended to provide a manufacturer-independent, objective evaluation of the "aberration-neutral" IOL's potential to increase patients' optical quality following lens replacement for cataract or refractive surgery.

\section{Methods}

This prospective laboratory analysis was performed at the David J Apple International Laboratory for Ocular Pathology, University Eye Hospital Heidelberg, Heidelberg, Germany.

The analysis involved two groups of hydrophilic, acrylic, copolymer, one-piece, monofocal IOLs. Group 1 included 10 spherical IOLs (C-flex $570 \mathrm{C}$ ) and Group 2 included 10 aspherical IOLs (C-flex $970 \mathrm{C}$ ). Both lens types are manufactured by Rayner Intraocular Lenses Ltd., Hove, UK and are based on the same body design, featuring an optic diameter of $5.75 \mathrm{~mm}$ with a sharp edge design and an overall diameter of $12 \mathrm{~mm}$. In this study, all analysed IOLs had a dioptric power of $26 \mathrm{D}$. The only difference between the IOLs was the SA value, resulting from slight differences in surface curvature.

The optical quality of all lenses was assessed using the OptiSpheric IOL PRO optical bench (Trioptics GmbH, Wedel, Germany) according to ISO 11979-2, Ophthalmic implants - Intraocular lenses - Part 2: Optical properties and test methods. This optical bench features different types of targets as objects, which are projected to the infinity through a collimator. As a result, the tested lens provides an image of the target at its focal plane. Composed of a microscope objective and an imaging system conjugated with a CCD camera, the measurement head scans through the imaging zone to find the best focus image created by the tested IOL. The image detected by the camera is then used for further analysis. The light source illuminating the target is a broad band visible spectrum light source associated with a narrow band interference filter at $546 \mathrm{~nm}$, in accordance with the ISO norm. Narrow band light is appropriate when comparing the two IOL models because they are made of the same material having the same chromatic aberration. The image obtained via the IOL is collected by a microscope and analysed by the integrated software. This method resonates with previous bench tests that have been used to analyse optical properties of different monofocal or multifocal aspheric IOLs [15-17].

In our setting, a thin line of light was chosen as a test target in order to obtain the Line Spread Function (LSF), from which the MTF and Strehl Ratio were calculated. The MTF of an optical system describes the amount of contrast that is passed through the system for a given spatial frequency or object size; it is defined as the amplitude of the image contrast divided by the amplitude of the object contrast and is a function of spatial frequency. The contrast decreases more rapidly at higher spatial frequencies (i.e. the number of line pairs [lp] per millimetre) or with object size. The MTF therefore represents the capability of an optical system to

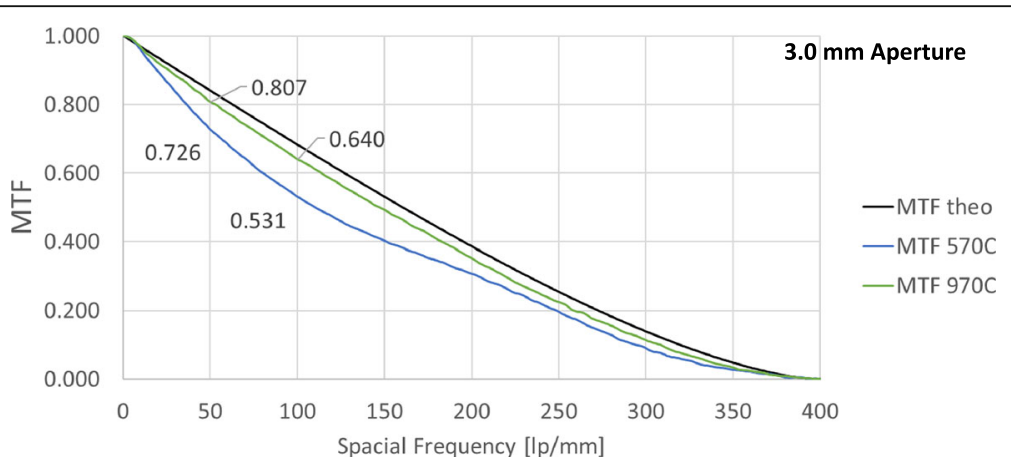

Fig. 1 Modulation Transfer Function: One example of each intraocular lens at $3.0 \mathrm{~mm}$ aperture. Black line: Theoretical upper limit, blue and green: Measured MTFs for both evaluated lenses 


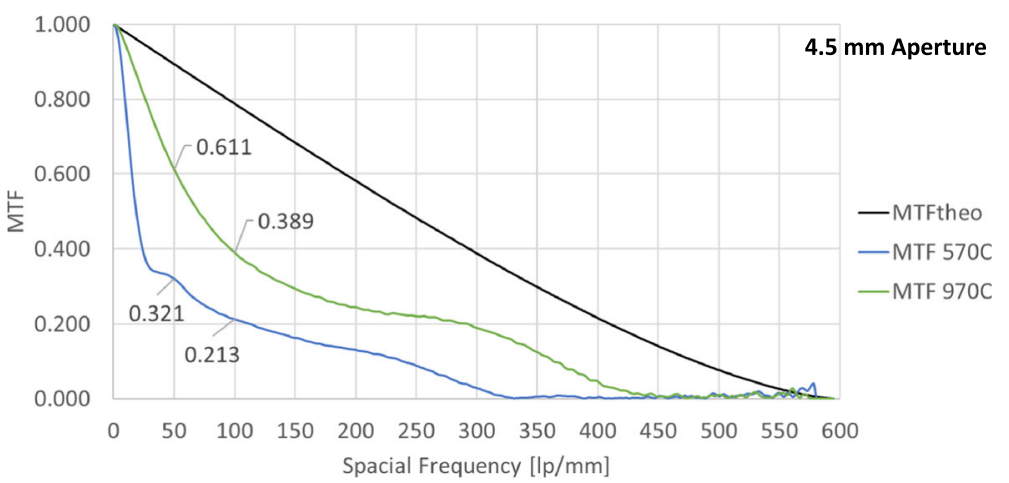

Fig. 2 Modulation Transfer Function: One example of each intraocular lens at $4.5 \mathrm{~mm}$ aperture. Black line: Theoretical upper limit, blue and green: Measured MTFs for both evaluated lenses

transfer the details of an object into an image. The Strehl Ratio enables the comparison of the maximum aberrated image intensity from a point source to the maximum achievable intensity using an ideal, diffractionlimited optical system. As such, the Strehl ratio is closely associated with quality of vision [18].

For testing, each IOL was placed within a model eye system. The haptics of the samples were rotationally oriented at random to compensate for the single slit measurement. The model cornea was an aberration-free achromatic doublet - as specified in ISO 11979-2 - so that any aberration observed or the effect of any aberration on image quality would result only from the IOL. In each instance, the IOL was placed in an IOL holder $(11.0-13.0 \mathrm{~mm})$ before being inserted into the model eye, which was filled with deionized water. Each IOL was positioned so that the lens's anterior side faced the incident light, and the IOL holder guaranteed tilt-free orientation of the lens during the testing procedure. The collimated light passing through the artificial cornea was focused on the IOL, thereby simulating the vergence of a human eye, and the device could automatically detect the optical axis of each IOL. Measurements were performed at ambient temperature as recommended by the ISO standard because the IOL dimensions do not deviate appreciably from those under in situ conditions.

The through-focus MTFs were measured using $3.0 \mathrm{~mm}$ and $4.5 \mathrm{~mm}$ apertures at $50 \mathrm{lp} / \mathrm{mm}$ and $100 \mathrm{lp} / \mathrm{mm}$ in the model eye. The focus was shifted gradually from an object at infinity to increasingly closer distances. The two apertures of $3.0 \mathrm{~mm}$ and $4.5 \mathrm{~mm}$ were chosen to correspond with photopic and mesopic surrounding conditions, while the spatial frequency of $50 \mathrm{lp} / \mathrm{mm}$ corresponded with the fundamental frequency of the 20/40 line on the Snellen eye chart. The Strehl Ratio was measured using the same $3.0 \mathrm{~mm}$ and $4.5 \mathrm{~mm}$ apertures.

Each lens underwent three measurement cycles without positional alteration to obtain all outcome metrics. This resulted in a mean value for each metric of each lens. Medians of the resulting ten mean values per group were then used for further statistical analysis. A MannWhitney $U$ test was performed to detect differences in optical property metrics between groups.

\section{Results}

Figures 1 and 2 show typical examples of the MTF values for $3.00 \mathrm{~mm}$ and $4.5 \mathrm{~mm}$ apertures at all spatial frequencies for one IOL per model. Tables 1 and 2 summarise the MTF results for $3 \mathrm{~mm}$ and $4.5 \mathrm{~mm}$ apertures at $50 \mathrm{lp} / \mathrm{mm}$ and $100 \mathrm{lp} / \mathrm{mm}$. The results demonstrate that for all measurements comparing the spherical and aspheric models, values for the aspheric IOL were always significantly higher $(p<0.01)$.

Tables 3 and 4 summarise the Strehl Ratio values for $3 \mathrm{~mm}$ and $4.5 \mathrm{~mm}$ apertures, which also show that values for the aspheric IOL were significantly higher in all instances $(p<0.01)$.

\section{Discussion}

Ocular SA has a significant influence on patients' quality of vision. Implanting specific aspheric lenses according to the intended optical use can help to optimise quality of vision and certain visual functions. Currently, there are two main approaches that are adhered to in practices. In the first approach (which has become widely accepted), an IOL with negative SA is selected in order to

Table 1 Modulation Transfer Function values at 3-mm aperture

\begin{tabular}{llll}
\hline MTF & Spherical IOL & Aspheric IOL & $P$-Value \\
\hline $50 \mathrm{lp} / \mathrm{mm}$ Median (Range) & $0.7133(0.6900$ to 0.7310$)$ & $0.8058(0.7970$ to 0.8130$)$ & $0.0002^{*}$ \\
$100 \mathrm{lp} / \mathrm{mm}$ Median (Range) & $0.5265(0.4980$ to 0.5430$)$ & $0.6345(0.6230$ to 0.6450) & $0.0002^{*}$ \\
\hline
\end{tabular}

*Statistically significant difference $(p<0.05$, Mann-Whitney- $U$ test) 
Table 2 Modulation Transfer Function (MTF) values at 4.5-mm aperture

\begin{tabular}{llll}
\hline MTF values & Spherical IOL & Aspheric IOL & $P$-Value \\
\hline Median (Range) at $50 \mathrm{Ip} / \mathrm{mm}$ & $0.2970(0.2790$ to 0.3280$)$ & $0.5935(0.5370$ to 0.6880$)$ & $<0.0001^{*}$ \\
Median (Range) at $100 \mathrm{lp} / \mathrm{mm}$ & $0.1975(0.1880$ to 0.2050$)$ & $0.3445(0.3230$ to 0.3920$)$ & $<0.0001^{*}$ \\
\hline
\end{tabular}

*Statistically significant difference $(p<0.05$, Mann-Whitney- $U$ test)

reduce overall ocular SA. Numerous studies prove the benefit of such IOLs for patients with large pupils, mainly at mesopic conditions [4, 5, 7]. The second approach entails the implantation of "aberration-neutral" IOLs, which are intended to avoid adding further SA to the existing ocular SA - namely that of the cornea [6].

In this study we have intended to provide an objective analysis of the potential optical benefit achievable with an "aberration-neutral" IOL. To exclude as many covariants as possible, we chose a study design which specifically examined IOLs developed by the same manufacturer, of the same material and of the same design, differing only in SA. Moreover, the study entailed a highly standardised analysis of optical properties, following the internationally-recognised ISO 11979-2 norm for testing optical systems.

The adopted model eye approach enabled a high level of control of the surrounding conditions: from corneal spherical aberrations to aperture sizes corresponding with pupil sizes at photopic and mesopic conditions to imaged spatial resolution, simulating tasks requiring different visual acuities. Consequently, this approach allowed us to show the tested IOLs' inherent ability to improve optical quality without other factors interfering with results (such as subjective perception). However, the aberration free model cornea of ISO 11979-2 is different from the mean human corneal SA, which is a limitation of this laboratory study.

The two main objective outcome metrics, the MTF and Strehl Ratio values, were chosen in accordance with the ISO 11979.2 norm. Optical bench evaluations, such as MTF testing, provide valuable information on the optical quality of IOLs. To accommodate varying surrounding conditions and to evaluate the possible optical benefits under these different conditions, we tested each IOL at $50 \mathrm{lp} / \mathrm{mm}$ and $100 \mathrm{lp} / \mathrm{mm}$ spatial resolutions (representing visual acuities of 20/40 and 20/20 respectively) and two aperture sizes $(3.0 \mathrm{~mm}$ and $4.5 \mathrm{~mm}$, representing photopic and mesopic surrounding conditions). The test conditions therefore represent clinically relevant visual tasks: seeing small details and seeing in mesopic conditions.

Overall, the MTF values were higher at all spatial resolutions for the aspheric IOL compared to the spherical IOL (Figs. 1 and 2). The aspheric IOL design achieved significantly higher MTF and Strehl Ratio values than the spherical design at the two selected and visually most relevant spatial resolutions and for both apertures. The differences between the aspheric and spherical design were more prominent in mesopic conditions. This suggests that patients with larger pupil sizes and/or working in mesopic conditions might benefit more from an aspheric IOL design than a spherical one. The larger difference in MTF at $4.5 \mathrm{~mm}$ pupil is expected from optical theory, since SA is highly aperture dependent. Our findings correspond with subjective evaluations of quality of vision after aspheric IOL implantation performed in previous studies $[5,6]$. However, these benefits are reported to become relevant in terms of quality of vision for pupil sizes of about $5 \mathrm{~mm}$ or larger only [5]. Our study was able to show significantly improved optical properties with the aspheric IOL when increasing the pupil size from $3.0 \mathrm{~mm}$ to $4.5 \mathrm{~mm}$ in comparison to the spherical IOL. The aspheric IOL also performed better than the spherical one under photopic conditions. These findings suggest that even patients with smaller pupil sizes, such as elderly cataract patients, might benefit from "aberration-neutral" aspheric IOLs, provided the lens has been chosen considering the existing corneal SA. However, individualising aspheric IOL selection to corneal SA is challenging, as several other factors are known to alter postoperative ocular SA. Amongst others, these contributing factors include surgically induced SA as a result of a change in corneal curvature due to the cataract incision as well as the fluctuations of SA measurements within same individual [14]. Various clinical studies have demonstrated little to no statistical significance between aspheric and spherical models of an IOL $[8,9]$.
Table 3 Strehl Ratio values for $3 \mathrm{~mm}$ aperture

\begin{tabular}{lccc}
\hline Strehl Ratio & Spherical IOL & Aspheric IOL & $P$-Value \\
\hline Median & 0.8143 & 0.9288 & $0.0002^{*}$ \\
Minimum & 0.7630 & 0.8800 & \\
Maximum & 0.8450 & 0.9410 & \\
\hline
\end{tabular}

*Statistically significant difference $(p<0.05$, Mann-Whitney- $U$ test)
Table 4 Strehl Ratio values for $4.5 \mathrm{~mm}$ aperture

\begin{tabular}{lccc}
\hline Strehl Ratio & Spherical IOL & Aspheric IOL & $P$-Value \\
\hline Median & 0.2355 & 0.4870 & $0.0002^{*}$ \\
Minimum & 0.2260 & 0.4430 & \\
Maximum & 0.2910 & 0.5080 & \\
\hline
\end{tabular}

*Statistically significant difference $(p<0.05$, Mann-Whitney- $U$ test) 


\section{Conclusions}

In summary, it can be concluded that patients with larger pupil sizes and/or working in mesopic conditions might benefit more from an aspheric IOL design. Additionally, patients with smaller pupils or individuals working in photopic conditions may yield better results in terms of quality of vision with an aspheric IOL design.

\section{Abbreviations}

IOL: Intraocular lens; IOLs: Intraocular lenses; LSF: Line Spread Function; MTF: Modulation Transfer Function; SA: Spherical aberration

\section{Acknowledgements}

We acknowledge the financial support of the Deutsche Forschungsgemeinschaft and Ruprecht-Karls-Universität Heidelberg within the funding programme Open Access Publishing.

\section{Funding}

This study was supported by the Klaus Tschira Stiftung. The IVCRC has received research grants, lecture fees, travel reimbursement from Rayner, UK. None of these funding bodies played a role in the design of the study and collection, analysis, and interpretation of data and in writing the manuscript.

\section{Availability of data and materials}

The datasets used and/or analysed during the current study available from the corresponding author on reasonable request.

\section{Authors' contributions}

$\Pi$ : conception and design, acquisition of data, analysis and interpretaion of data, drafting and revising the manuscript. GUA: analysis and interpretaion of data, revising the manuscript, final approval. CYC: participation in study design, analysis and interpretaion of data, revising the manuscript. SL: acquisition and statistical analysis of data, revising the manuscript. CM: analysis and interpretaion of data, revising the manuscript. RK: conception and design, analysis and interpretaion of data, revising the manuscript, final approval. All authors read and approved the final manuscript.

\section{Competing interests}

The IVCRC has received research grants, lecture fees, travel reimbursement from Rayner, UK.

\section{Consent for publication}

Not applicable.

\section{Ethics approval and consent to participate}

This study did not involve human participants, human data, or human tissue requiring need to include a statement on ethics approval and consent.

\footnotetext{
Author details

'David J Apple International Laboratory for Ocular Pathology and International Vision Correction Research Centre (IVCRC), Department of Ophthalmology, University of Heidelberg, Im Neuenheimer Feld 400, Heidelberg 69120, Germany. ${ }^{2}$ Department of Ophthalmology, Kangbuk Samsung Hospital, Sungkyunkwan University School of Medicine, Kangbuk Samsung Hospital, Pyeong-dong, Jongno-gu, Seoul, South Korea. ${ }^{3}$ Eye Clinic, Klinikum rechts der Isar der Technischen Universität München, Munich, Germany.
}

Received: 5 November 2016 Accepted: 27 January 2017

Published online: 08 February 2017

\section{References}

1. Wang L, Dai E, Koch DD, Nathoo A. Optical aberrations of the human anterior cornea. J Cataract Refract Surg. 2003;29:1514-21.

2. Wang L, Santaella RM, Booth $M$, Koch DD. Higher-order aberrations from the internal optics of the eye. J Cataract Refract Surg. 2005;31:1512-9.
3. de Sanctis U, Vinai L, Bartoli E, Donna P, Grignolo F. Total spherical aberration of the cornea in patients with cataract. Optom Vis Sci. 2014;91:1251-8

4. Montés-Micó R, Ferrer-Blasco T, Cerviño A. Analysis of the possible benefits of aspheric intraocular lenses: review of the literature. J Cataract Refract Surg. 2009;35:172-81.

5. Kohnen T, Klaproth OK, Bühren J. Effect of intraocular lens asphericity on quality of vision after cataract removal: an intraindividual comparison. Ophthalmology. 2009;116:1697-706.

6. Khoramnia R, Fitting A, Rabsilber TM, Thomas BC, Auffarth GU, Holzer MP. [Functional results after implantation of an aspheric, aberration-neutral intraocular lens]. Klin Monbl Augenheilkd. 2015;232:181-8.

7. Schuster AK, Tesarz J, Vossmerbaeumer U. Ocular wavefront analysis of aspheric compared with spherical monofocal intraocular lenses in cataract surgery: Systematic review with metaanalysis. J Cataract Refract Surg. 2015;41:1088-97.

8. Kasper T, Bühren J, Kohnen T. Visual performance of aspherical and spherical intraocular lenses: intraindividual comparison of visual acuity, contrast sensitivity, and higher-order aberrations. J Cataract Refract Surg. 2006:32:2022-9.

9. Kurz S, Krummenauer F, Thieme H, Dick HB. Contrast sensitivity after implantation of a spherical versus an aspherical intraocular lens in biaxial microincision cataract surgery. J Cataract Refract Surg. 2007;33:393-400.

10. Wang L, Koch DD. Custom optimization of intraocular lens asphericity. J Cataract Refract Surg. 2007;33:1713-20.

11. Kohnen T, Klaproth OK. [Aspheric intraocular lenses]. Ophthalmologe. 2008;105:234-40.

12. Kretz FTA, Son H, Liebing S, Tandogan T, Auffarth GU. [Impact of an Aspherical Aberration Correcting Monofocal Intraocular Lens on Patient Satisfaction for Daily Life Activities: The Heidelberg Daily Task Evaluation (DATE) Questionnaire]. Klin Monbl Augenheilkd. 2015;232:940-6.

13. Kretz FTA, Tandogan T, Khoramnia R, Auffarth GU. High order aberration and straylight evaluation after cataract surgery with implantation of an aspheric, aberration correcting monofocal intraocular lens. Int J Ophthalmol. 2015;8:736-41.

14. Negishi K, Kodama C, Yamaguchi T, Torii H, Saiki M, Dogru M, et al. Predictability of ocular spherical aberration after cataract surgery determined using preoperative corneal spherical aberration. J Cataract Refract Surg. 2010;36:756-61.

15. Pieh S, Fiala W, Malz A, Stork W. In vitro strehl ratios with spherical, aberration-free, average, and customized spherical aberration-correcting intraocular lenses. Invest Ophthalmol Vis Sci. 2009;50:1264-70.

16. Gatinel D, Houbrechts Y. Comparison of bifocal and trifocal diffractive and refractive intraocular lenses using an optical bench. J Cataract Refract Surg. 2013;39:1093-9.

17. Tandogan T, Khoramnia R, Choi CY, Scheuerle A, Wenzel M, Hugger P, et al. Optical and material analysis of opacified hydrophilic intraocular lenses after explantation: a laboratory study. BMC Ophthalmol. 2015;15:170.

18. Marsack JD, Thibos LN, Applegate RA. Metrics of optical quality derived from wave aberrations predict visual performance. J Vis. 2004:4:322-8. 